\title{
Species-specific recognition of beetle cues by the nematode Pristionchus maupasi
}

\author{
Ray L. Hong, ${ }^{a}$ Aleš Svatoš, ${ }^{b}$ Matthias Herrmann, ${ }^{a}$ and Ralf J. Sommera,* \\ ${ }^{a}$ Department for Evolutionary Biology, Max-Planck Institute for Developmental Biology, Tuebingen, Germany \\ ${ }^{b}$ Max-Planck Institute for Chemical Ecology, Mass Spectrometry Research Group, Jena, Germany \\ *Author for correspondence (email: ralf.sommer@tuebingen.mpg.de)
}

\begin{abstract}
SUMMARY The environment has a strong effect on development as is best seen in the various examples of phenotypic plasticity. Besides abiotic factors, the interactions between organisms are part of the adaptive forces shaping the evolution of species. To study how ecology influences development, model organisms have to be investigated in their environmental context. We have recently shown that the nematode Pristionchus pacificus and its relatives are closely associated with scarab beetles with a high degree of species specificity. For example, $P$. pacificus is associated with the oriental beetle Exomala orientalis in Japan and the northeastern United States, whereas Pristionchus maupasi is primarily isolated from cockchafers of the genus Melolontha in Europe. Here, we investigate how Pristionchus nematodes identify their specific insect hosts by using chemotaxis
\end{abstract}

studies originally established in Caenorhabditis elegans. We observed that $P$. maupasi is exclusively attracted to phenol, one of the sex attractants of Melolontha beetles, and that attraction was also observed when washes of adult beetles were used instead of pure compounds. Furthermore, $P$. maupasi chemoattraction to phenol synergizes with plant volatiles such as the green leaf alcohol and linalool, demonstrating that nematodes can integrate distinct chemical senses from multiple trophic levels. In contrast, another cockchafer-associated nematode, Diplogasteriodes magnus, was not strongly attracted to phenol. We conclude that interception of the insect communication system might be a recurring strategy of Pristionchus nematodes but that different nematodes use distinct chemical cues for finding their beetle hosts.

\section{INTRODUCTION}

Evolutionary developmental biology seeks to unite the molecular genetic approach of development with evolutionary biology (Rudel and Sommer 2003). However, a fully integrated approach requires the incorporation of yet another discipline, namely ecology (Gilbert and Bolker 2003). Van Valen once suggested that "evolution is the control of development by ecology" (Van Valen 1973). The most prominent examples are the many cases of phenotypic plasticity, which highlight the importance of ecology for development and evolution (Callahan et al. 1997; West-Eberhard 2005). Thus, there is an obvious need for interdisciplinary studies to confront certain realities of ecology and its effects on development.

Such integrative studies can be potentially achieved with nematodes of the genus Pristionchus. Pristionchus pacificus has been established as a satellite organism in evolutionary developmental biology, in particular in comparing vulva patterning between $P$. pacificus and Caenorhabditis elegans (Zheng et al. 2005; Schlager et al. 2006; Hong and Sommer 2006b). Field studies in Europe, North America, and Asia revealed that Pristionchus nematodes are associated with scarab beetles in an almost species-specific manner (Herrmann et al. 2006a, b, 2007; Mayer et al. 2007). For example, P. pacificus is associated with the oriental beetle Exomala orientalis in Japan and in the northeastern United States (Herrmann et al. 2007). In western Europe, Pristionchus maupasi and P. entomophagus are two widespread hermaphroditic species that are associated with the cockchafers Melolontha ssp. and the dung beetles Geotrupes ssp., respectively (Herrmann et al. 2006a).

Although the nematode-beetle interactions are complex, they can also be surprisingly specific. A prime example of the species-specific necromenic association is $P$. maupasi and its primary host, the European cockchafers of the genus Melolontha. Although P. maupasi has been repeatedly isolated as the primary Pristionchus nematode associated with adult cockchafers during swarming (spring 2005-2007, Herrmann et al. 2006a and unpublished results), P. maupasi populations have also been isolated from earlier developmental stages such as grubs, pupae, and eclosed adults. At the same time, cockchafers also host a distantly related nematode, Diplogasteroides magnus, which can co-occur with P. maupasi on the same beetles (Herrmann et al. 2006a; Kiontke et al. 2001). Thus, cockchafers represent a microcosm for certain nematodes, but how such host preference is achieved remains completely unknown. 
The interaction with host insects forms the basis for Pristionchus ecology. This nematode-insect association, in which nematodes embark on the insects and wait for their hosts to die before resuming development on the cadaver, is known as necromeny and represents one of the two most important types of nematode-invertebrate interactions (Kiontke and Fitch 2006). Pristionchus nematodes infest live insects but do not noticeably reduce the fitness of their hosts; instead they likely consume the microorganisms on the decomposing insect carcasses. At the individual level, several critical developmental decisions such as dauer larvae formation, mouth form polyphenism, and reproductive transition can be influenced by changes in temperature and food on the host. Hence, the developmental state and intrinsic temporal differences among different host species are important considerations. The outcomes of these developmental interactions with the host ecology can in turn affect population size and gene flow. Therefore, we hope to delineate a more accurate portrayal of Pristionchus host environment by investigating into the biotic factors that modulate host preference.

We used chemotaxis studies to obtain insights into the ecological associations of Pristionchus nematodes. Chemotaxis studies under laboratory conditions have long been used in C. elegans to study various aspects of the behavior, development, and neurobiology of nematodes in general (Bargmann 2006). It was initially surprising to find that four representative Pristionchus species could be distinguished by their chemoattractive profiles (Hong and Sommer 2006a). These studies also provided the first evidence that Pristionchus nematodes are attracted to certain insect sex pheromones. For example, the oriental beetle associated P. pacificus is strongly attracted to this beetle's sex pheromone, $(Z)$-7-tetradecen-2one under laboratory conditions in a species-specific manner (Herrmann et al. 2007). These results suggested that interception of the chemical communication system of the insect is likely to be involved in host preferences.

To determine whether $P$. pacificus' specific attraction toward the pheromone of its preferred host represents a general mechanism for Pristionchus nematodes, we performed chemotaxis studies on the cockchafer-associated $P$. maupasi using pure chemical compounds as well as cuticular washes from various developmental stages of cockchafers. These studies provide a more detailed approximation of Pristionchus ecology necessary for understanding Pristionchus developmental biology in their evolutionary context.

\section{MATERIALS AND METHODS}

\section{Nematode strains}

Pristionchus nematode maintenance is the same as for C. elegans. P. maupasi RS5015 and D. magnus RS1986 were originally isolated from Melolontha beetles and have been maintained on Escherichia coli OP50 and inbred for more than 2 years (Herrmann et al. 2006a). They show no gross developmental or genetic variations. Along with RS5015, other homogeneous laboratory populations used for comparative analyses are $P$. pacificus PS1843, $P$. entomophagus RS144, P. uniformis RS5167, C. elegans N2, and Poikilolaimus oxycercus $\mathrm{RS} 2045$. All nematode species have a similar generation time of 3-6 days at $20^{\circ} \mathrm{C}$, except for $P$. oxycercus, which has a generation time of $\sim 3$ weeks.

\section{Beetle washes}

Feeding virgin adult Melolontha hippocastani were collected in Mannheim, Germany in May 2006, and kept at $4^{\circ} \mathrm{C}$ up to 2 weeks before washing. Attraction strengths decreased from samples that were kept longer. Three feeding adult individuals of the same sex were soaked in 6-8 $\mathrm{ml}$ of dichloromethane $\left(\mathrm{CH}_{2} \mathrm{Cl}_{2}\right)$ in a glass tube for $24 \mathrm{~h}$ at $23^{\circ} \mathrm{C}$. The washes were then vacuum dried at $30^{\circ} \mathrm{C}$ in small glass sample tubes, and resuspended in $120 \mu$ of pure ethanol. Two washes of each sex were processed this way and used for chemoattraction assays. Dichloromethane without beetle specimen processed the same way served as the counter-attractant control. Larger scale preparations for gas chromatography and mass spectrometry (GC-MS) were prepared similarly with 128 males and 139 females. We surmised that treating adults in groups better approximate their behavior in nature as swarms. Pupae were collected from soil in Karlsruhe, Germany in August 2006 and washed immediately. Other pupae were kept in soil for 2 weeks to obtain newly eclosed adults before feeding. For pupae and eclosed adult washes, only a single beetle was processed per glass tube, and the washes used for the chemoattraction assays represent at least three individuals of each sex. The sexes of pupae and adults were determined by the larger antennae of male cockchafers compared with those of females.

\section{Nematode chemotaxis assays}

Population chemotaxis assays for Pristionchus species were modified from that used on C. elegans as described previously (Bargmann et al. 1993; Hong and Sommer 2006a; Herrmann et al. 2007). In brief, the chemotaxis index was calculated as: (Number of animals at attractant - Number of animals at counter attractant)/(Total number of worms at both attractant and counter attractant). We used animals from 5-day-old cultures that were started with 10 gravid females or hermaphrodites grown at $20^{\circ} \mathrm{C}$ to conduct the chemotaxis assay. In those cultures, $>80 \%$ of the populations were adults.

Worms were washed three times in M9 buffer, briefly centrifuged in $15 \mathrm{ml}$ polypropylene tubes, and loaded onto $8.5 \mathrm{~cm}$ round assay plates containing $0.5 \mathrm{~cm}$ thick NGM agar. $1.5 \mu \mathrm{l}$ of attractant/counter attractant and $1 \mathrm{M}$ sodium azide were used and animals within $\mathrm{a} \sim 0.5 \mathrm{~cm}$ radius circle around each point odor source were counted. All attractive responses described were summarized from at least 10 replicates of $10 \mathrm{~J} 3$-adult stage individuals (average 30) over two to three separate experiments from multiple culturing plates. Because the nematode strains are near isogenic, we did not observe dramatic chemoattraction differences for the same compound between separate experiments. Peak responses were initially scored at 2, 5, and $\sim 15 \mathrm{~h}$ intervals. We interpret those compounds with $95 \%$ confidence intervals intersecting zero as neutral responses, because nematodes can randomly bias one side of the plate versus the other when there is no attraction. Final values for a 
given species always were taken at the same time point with the highest absolute chemoattraction index (CI). In general, D. magnus peak responses occur between 2 and $9 \mathrm{~h}$, whereas peak responses of Pristionchus, C. elegans, and P. oxycercus species were reached between 1 and $15 \mathrm{~h}$.

\section{Chemicals}

Phenol crystals (Roth, Karlsruhe, Germany) were diluted in 100\% ethanol to $0.1-10 \%(\mathrm{w} / \mathrm{v})$ as stock solution. Other compounds were purchased from Sigma-Aldrich ( $>97 \%$ purity; St. Louis, MO, USA) and likewise diluted in ethanol to $0.1-10 \%(\mathrm{v} / \mathrm{v})$. For double component blends of phenol and plant volatiles, $0.75 \mathrm{nl}$ phenol was mixed with $75 \mathrm{nl}$ of green leaf alcohol ((Z)-3-hexen-1-ol [GLA]) or $7.5 \mathrm{nl}$ of linalool. Thus, in blends with three components, $1 / 3$ of the amount used in single component assays was used in the mixtures. In a slightly different setup, a blend containing $0.75 \mu 1$ of a newly eclosed adult female wash with $15 \mathrm{nl}$ linalool was compared with $0.75 \mu \mathrm{l}$ of the wash or $15 \mathrm{nl}$ linalool alone. To minimize batch variations, we always conducted single to blended comparisons concurrently using the same batch of worms.

Error bars denote standard error of the mean. Student's twotailed $t$-test was done in Microsoft Excel and single factor ANOVA done in Excel or STATA (College Station, TX, USA) with Bonferroni's correction $(\alpha=0.05)$.

\section{GC-MS}

GC-MS analyses were performed with a HP6890 (Hewlett Packard, Santa Clara, CA, USA) gas chromatograph interfaced to a MasSpec 2 (Waters Micromass UK Ltd., Milford, MA, USA) in positive ion mode using $70 \mathrm{eV}$ ionization energy. Separation was achieved on a J\&W Scientific (Folsom, CA, USA) DB-5 capillary column ( $30 \mathrm{~m}$, ID $0.25 \mathrm{~mm}, 0.25 \mu \mathrm{m}$ film thickness) using helium at constant flow as carrier gas $(0.6 \mathrm{ml} / \mathrm{min})$. Samples in hexane were injected splitless $(1$ or $3 \mu \mathrm{l})$ at injector temperature $220^{\circ} \mathrm{C}$. Temperature program used: $40^{\circ} \mathrm{C}$ for $2 \mathrm{~min}$, then it was heated to $140^{\circ} \mathrm{C}$ by $70^{\circ} \mathrm{C} / \mathrm{min}$, to $240^{\circ} \mathrm{C}$ by $2^{\circ} \mathrm{C} / \mathrm{min}$, to $300^{\circ} \mathrm{C}$ at $20^{\circ} \mathrm{C} / \mathrm{min}$ gradients and the final temperature was held for $10 \mathrm{~min}$. The transfer line from GC to EI-MS source was heated at $280^{\circ} \mathrm{C}$. The compounds were identified based on their EI-MS fragmentation patterns using fragmentation rules and with mass spectral databases (NIST 02 and Wiley v. 7).

\section{RESULTS}

\section{Phenol is a strong species-specific attractant for P. maupasi}

Previous studies indicated that four closely related Pristionchus species associated with beetles have distinct chemotaxis profiles toward plant and insect compounds (Hong and Sommer 2006a). In particular, $P$. pacificus is strongly attracted to the sex pheromone of the oriental beetle, $(Z)-7$ tetradecen-2-one under laboratory conditions (Herrmann et al. 2007). To test if compounds from Melolontha spp. could also be used by $P$. maupasi nematodes to locate its preferred hosts, we performed chemotaxis assays using phe- nol, the known Melolontha sex attractant, as an attractant (Ruther et al. 2002b) (Fig. 1A). We found that phenol is attractive over a 100 -fold range to only $P$. maupasi but not to other nematodes. Using between 15 and $150 \mathrm{nl}$ of phenol, the CI reached 0.6 and 0.7 , respectively, which represents the highest $\mathrm{CI}$ in $P$. maupasi for any compound tested. To further determine the species specificity of the response to phenol, we tested six additional nematode species representing four different clades. Closely related species such as $P$. pacificus did not respond to phenol, whereas the dung beetle-associated $P$. entomophagus and the Colorado potato beetle-associated P. uniformis avoided phenol altogether at 15-150 nl (Fig. 1A). Avoidance behavior represents odor recognition and may
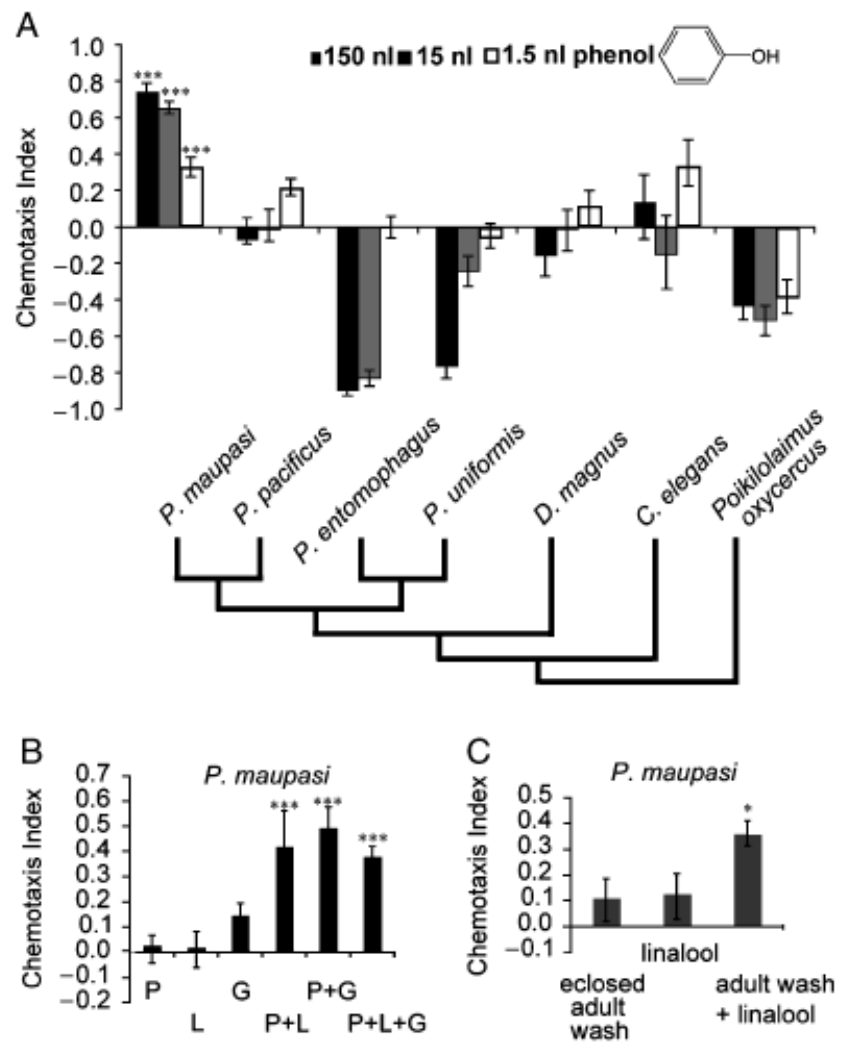

Fig. 1. Pristionchus maupasi attraction to phenol and blends and phylogenetic relationship among species. (A) P. maupasi is specifically attracted to $1.5-150 \mathrm{nl}$ phenol compared with other nematodes (ANOVA ${ }^{* * *} P<0.001$, except for Pristionchus pacificus and Diplogasteriodes magnus responses to $1.5 \mathrm{nl}$ phenol, whereby $P>0.05$ and $P<0.05$, respectively). The phylogeny of Pristionchus species is modified from Mayer et al. (2007). (B) Synergy of the phenol attractant and plant volatiles. P, phenol; L, linalool; $\mathrm{G}$, green leaf alcohol. Note that up to 3-fold less phenol was used than the lowest amount shown in Fig. 2A. (C) Synergy of newly eclosed female adult wash and linalool (ANOVA, ${ }^{*} P=0.05$ ). Significant differences are shown by one-way ANOVA with Bonferroni's correction of blends to constituent compounds *** $P<0.001$, except $\mathrm{P}+\mathrm{G}$ by $\mathrm{G}$ alone, $P=0.006$, and $\mathrm{P}+\mathrm{G}+\mathrm{L}$ by $\mathrm{P}$ alone, $P=0.14$. Error bars denote standard error of the mean and each bar represents $10-20$ replicates from $\geq 2$ separate experiments. 
promote host recognition among closely related Pristionchus species. These results suggest that nematodes may utilize both attraction and repulsion behavior to execute their habitat preferences. Furthermore, phenol recognition and chemotaxis response to phenol vary between nematode species. In particular, the behavioral response to phenol may have changed multiple times independently.

\section{Plant compounds synergize with phenol}

Because pure compounds are rarely encountered in nature, we sought to identify additional accessory compounds that can synergize with phenol. One class of compounds likely to cooccur with the presence of cockchafers is plant defense volatiles, which are upregulated as defense agents when these phytophagus adult beetles feed on leaves. Cockchafers in Germany feed on Quercus rubra (oak), Prunus serotina (black cherry), and Carpinus betulus (hornbeam) and male cockchafers have been shown to be attracted to volatiles from these trees containing leaf aldehydes and the GLA (Ruther et al. 2000). GLA is also a plant kairomone that enhances the attractiveness of phenol in field tests (Reinecke et al. 2002; Ruther et al. 2002a). Hence plant defense volatiles are an integral part of the cockchafer's sexual communication system and candidates for nematode attraction. The beetles themselves are also a minor source of plant volatiles ingested from their diet. We found that both GLA and another plant defense compound linalool can synergize with phenol up to $\sim 40$-fold increase, indicating that $P$. maupasi is able to integrate odor signals across multiple trophic levels (Fig. 1B). Thus, the addition of plant volatiles to phenol may be a critical contextual cue for announcing the feeding and sexual maturation of cockchafers to the nematodes.

\section{$\boldsymbol{P}$. maupasi is attracted by cuticular beetle washes}

Cockchafers have a generation time of 3-4 years, with nonfeeding adults overwintering in pupal chambers underground. To better describe nematode-beetle interactions in the context of the environment, we washed beetles isolated from the wild with dichloromethane and used different developmental stages of the insect in these assays. We asked which of these cuticular washes, representing the beetles' odor profiles at

Fig. 2. Nematode attraction toward dichloromethane washes of Melolontha hippocastani beetles. (A) Washes of male and female feeding adults were most attractive to Pristionchus maupasi, compared with closely related Pristionchus species. (B) Washes from pupae and newly emerged adults were less attractive to $P$. maupasi than feeding adults (same data as (A) for feeding adults). (C) Diplogasteriodes magnus attraction toward the three developmental stages of cockchafers showed no significant difference to each other. Error bars denote standard error of the mean and each bar represents $10-15$ replicates from $\geq 2$ separate experiments. Single factor ANOVA $* P<0.05,{ }^{* * *} P<0.001$. advanced stages of their life cycle, are specifically attractive to $P$. maupasi. Figure 2A shows that feeding adult cockchafers washes from both sexes were the most attractive to $P$. maupasi

male wash $\mathbf{\square}$ female wash
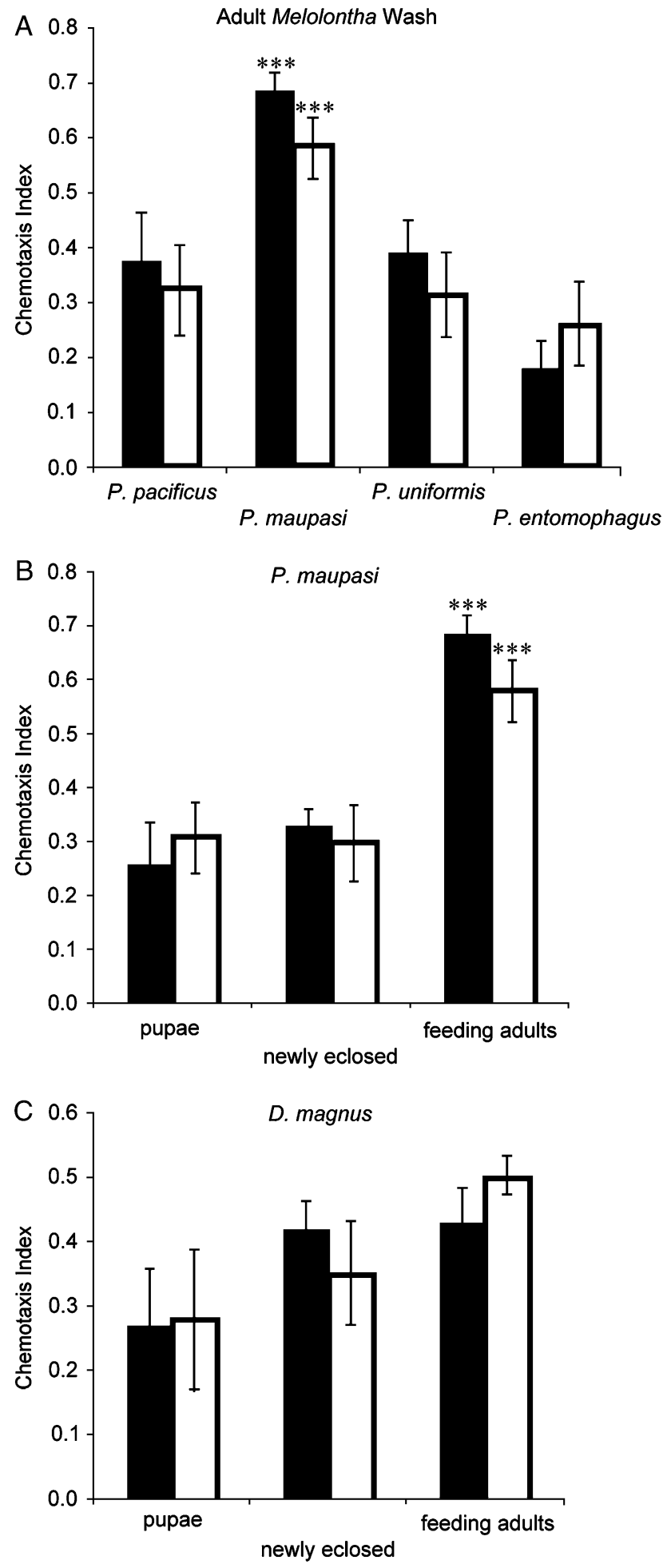
compared with closely related Pristionchus species that are associated with other beetles. Washes from two earlier developmental stages of cockchafers as pupae and non-feeding adult washes were significantly less attractive to $P$. maupasi than feeding adult washes (Fig. 2B). These washes were even less attractive to the other three Pristionchus species (data not shown). No significant difference between washes from female and male beetles at any developmental stage tested were found. To confirm that phenol is present in the cuticular washes of Melolontha beetles, we performed GC-MS analyses on the wash from feeding adults of both sexes and identified phenol, along with several other compounds (data not shown). This finding is consistent with previous studies of European cockchafers (Ruther et al. 2001). We conclude that the most specific attractive stage for $P$. maupasi infestation seems to be the phenol-producing virgin adults of both sexes that have just initiated feeding on the spring leaves.

To further demonstrate the principle that plant volatiles enhanced the attractiveness of beetles, we mixed the plant volatile linalool with the wash of newly eclosed adults just emerging out of their pupal cases. We found that the presence of linalool can enhance the attractiveness of a complex blend, the weakly attractive pre-feeding adult stage (Fig. 1C). Linalool, however, did not enhance the attraction of the pupae wash (data not shown). This result further affirms our model that cockchafers feeding may be crucial for transforming weakly attractive eclosed adults to attractive host targets.

\section{D. magnus has a different attraction profile}

Finally, we wanted to know if different nematodes that occupy the same beetle as ecological niche have similar olfaction profiles. Therefore, we tested D. magnus, the species that is the most common nematode observed on cockchafers (Herrmann et al. 2006a; Kiontke et al. 2001). In contrast to $P$. maupasi, D. magnus showed moderate attraction to all three developmental washes (Fig. 2C). Although both $P$. maupasi and D. magnus belong to the family Diplogastridae, D. magnus was not attracted to phenol (Fig 1A), but more attracted than P. maupasi to GLA and linalool (using $15 \mathrm{nl}$, data not shown). These results suggest that different nematode species associated with the same insect can have different host recognition strategies.

\section{DISCUSSION}

Numerous necromenic nematodes are closely associated with insects but their insect preference and utilization of host cues have never been investigated in detail (Poinar 1972; Kiontke and Fitch 2006). For example, Caenorhabditis drosophilae is found regularly on Drosophila nigrospiracula (Diptera, Drosophilidae) near decomposing saguaro cacti in Arizona (Kiontke 1997), whereas Caenorhabditis japonica has been isolated from the nest of the adult shield bug Parastrachia japonensis (Heteroptera, Cydnidae) in Japan (Kiontke et al. 2002). Unlike entomopathogenic nematodes, which parasitize and kill their hosts and are often generalists with a broad host range, necromenic nematodes are usually associated with only few specific insects. Here we investigated if necromenic species in the genus Pristionchus use specific insect cues to locate their preferred hosts.

We identified the cockchafer-specific host cue phenol and demonstrated its ability to synergize with plant volatiles - the first time to our knowledge where nematodes are shown to integrate distinct chemical sources from multiple trophic levels. In addition, P. maupasi was also specifically attracted to the cuticular washes of adult Melolontha beetles containing complex bouquets of environmentally informative compounds, including phenol. We initially did not expect a soildwelling nematode to use leaf volatiles to locate adult beetle hosts, which spend part of their time on the arial part of trees. However, it is well known that feeding adult cockchafers, including gravid females, move back and forth multiple times between the soil and trees, as well as spend a significant proportion of their time sitting rather than swarming (Balachowsky 1962; Schwenke 1974; Ruther et al. 2002b). It should be further noted that feeding adults are relatively short lived, usually around 4 weeks, whereas grubs and pupae stages can last for up to 3 years, with the eclosed adults also overwintering underground. Therefore, feeding adult beetles seem to be the most attractive to a nematode with a necromenic life history. The P. maupasi attraction profiles coincide with the most dispersive stage of cockchafers, as well as the most mobile phase for transport of the nematode. Given the specific life cycle of the European cockchafer, we hope to investigate the duration of Pristionchus populations on different developmental stages of host beetles in the future.

The results presented in this study, in combination with the previous finding that $P$. pacificus is specifically attracted to the sex pheromone of the oriental beetle (Herrmann et al. 2007), raises the possibility that Pristionchus nematodes recognize the species-specific communication of insects related to their ecology. Previous studies on the role of plant and beetle host compounds on nematode chemotaxis behavior have focused on entomopathogenic nematodes (Ishikawa et al. 1986; Shuto and Watanabe 1987; Stamps and Linit 1998, 2001; Rasmann et al. 2005). However, these studies did not investigate the species specificity of interactions and their potential mechanisms.

We isolated phenol from both sexes, although phenol has been referred to as a sex attractant commonly found only in female beetles. Although it is possible that physical contact between individual beetles before or during the collection process could have distributed phenol between the sexes, it should be noted that earlier studies of phenol extracted from 
two species of Melolonthine beetles did not explicitly investigate males (Henzell and Lowe 1970; Ruther et al. 2001). Recent studies in Drosophila melanogaster supports the notion that specific cuticular lipids from males can be a generalizing cue for sexual maturation in both sexes, with both attractive and aversive functions (Ejima et al. 2007). In addition to being a sex attractant, phenol may also function as an antimicrobial agent and as such is likely to be present in both sexes. Hence it has been proposed that phenol may be selected initially during evolution for its antimicrobial property and only later became co-opted as a sex pheromone (Leal 1997).

Differences in the chemotaxis behavior of $P$. maupasi and D. magnus correlate with differences in their beetle association. First, D. magnus associates equally with all developmental stages of the beetle (at least at our limited collection sites). Second, after the artificial death of the insect under laboratory condition, there is a succession of the occurrence of adult nematodes on agar plates: $D$. magnus is observed first after a few days, whereas $P$. maupasi is often only seen after 10 days or more (Herrmann et al. 2006a). However, the ecological significance of this observation awaits further analysis. Also, future studies will have to address which cues mediate the association of D. magnus with Melolontha beetles.

The specific interception of the beetle communication system by Pristionchus nematodes represents an important entry point for the interdisciplinary analysis of ecological developmental biology in nematodes. Future genetic studies can reveal which genetic and molecular mechanisms Pristionchus nematodes use to recognize the insect sex pheromones and the type of natural variation responsible for the adaptation of the nematode-beetle association. In the long run, the detailed knowledge about the Pristionchus ecology is a helpful approximation toward the integration of ecology, development, and evolution.

\section{Acknowledgments}

We thank members of the Sommer lab for discussion and critically reading the manuscript. R. L. H. was supported by the Alexander von Humboldt Postdoctoral Fellowship.

\section{REFERENCES}

Balachowsky, A. S. 1962. Entomologie Appliquee A L'Agriculture. Masson, Paris.

Bargmann, C. I. 2006. Comparative chemosensation from receptors to ecology. Nature 444: 295-301.

Bargmann, C. I., Hartwieg, E., and Horvitz, H. R. 1993. Odorant-selective genes and neurons mediate olfaction in C. elegans. Cell 74: 515-527.

Callahan, H. S., Pigliucci, M., and Schlichting, C. D. 1997. Developmental phenotypic plasticity: where ecology and evolution meet molecular biology. Bioessays 19: 519-525.

Ejima, A., et al. 2007. Generalization of courtship learning in Drosophila is mediated by cis-vaccenyl acetate. Curr. Biol. 17: 599-605.

Gilbert, S. F., and Bolker, J. A. 2003. Ecological developmental biology: preface to the symposium. Evol. Dev. 5: 3-8.
Henzell, R. F., and Lowe, M. D. 1970. Sex attractant of the grass grub beetle. Science 168: 1005-1006.

Herrmann, M., Mayer, W., Hong, R. L., Kienle, S., Minasaki, R., and Sommer, R. J. 2007. The nematode Pristionchus pacificus (Nematoda: Diplogastridae) is associated with the Oriental beetle Exomala orientalis (Coleoptera: Scarabaeidae) in Japan. Zool. Sci. 24: 883-889.

Herrmann, M., Mayer, W. E., and Sommer, R. J. 2006a. Nematodes of the genus Pristionchus are closely associated with scarab beetles and the Colorado potato beetle in Western Europe. Zoology 109: 96-108.

Herrmann, M., Mayer, W. E., and Sommer, R. J. 2006b. Sex, bugs and Haldane's rule: the nematode genus Pristionchus in the United States. Front. Zool. 3: 14.

Hong, R. L., and Sommer, R. J. 2006a. Chemoattraction in Pristionchus nematodes and implications for insect recognition. Curr. Biol. 16: 23592365.

Hong, R. L., and Sommer, R. J. 2006b. Pristionchus pacificus: a wellrounded nematode. Bioessays 28: 651-659.

Ishikawa, M., Shuto, Y., and Watanabe, H. 1986. Beta-Myrcene, a potent attractant component of pine wood for the pine wood nematode, Bursaphelenchus xylophilus. Agric. Biol. Chem. 50: 1863-1866.

Kiontke, K. 1997. Description of Rhabditis (Caenorhabditis) drosophilae $\mathrm{n}$ sp and $R$. (C) sonorae $\mathrm{n}$ sp (Nematoda: Rhabditida) from saguaro cactus rot in Arizona. Fundam. Appl. Nematol. 20: 305-315.

Kiontke, K., and Fitch, D. 2006. The phylogenetic relationships of Caenorhabditis and other rhabditids; wormbook. ed, The C. elegans Research Community, Wormbook; doi:/10.1895/wormbook.1.11.1, http:// www.wormbook.org

Kiontke, K., Hironaka, M., and Sudhaus, W. 2002. Description of $\mathrm{Ca}$ enorhabditis japonica $\mathrm{n}$. sp (Nematoda: Rhabditida) associated with the burrower bug Parastrachia japonensis (Heteroptera: Cydnidae) in Japan. Nematology 4: 933-941.

Kiontke, K., Manegold, A., and Sudhaus, W. 2001. Redescription of Diplogasteroides nasuensis Takaki, 1941 and D. magnus Volk, 1950 (Nematoda: Diplogastrina) associated with Scarabaeidae (Coleoptera). Nematology 3: 817-832.

Leal, W. S. 1997. Evolution of sex pheromone communication in plant feeding scarab beetles. Carde, R. T. and Minks, A. K. (eds.) Pheromone Research: New Directions. Chapman \& Hall, New York, pp. 505-513.

Mayer, W. E., Herrmann, M., and Sommer, R. J. 2007. Phylogeny of the nematode genus Pristionchus and implications for biodiversity, biogeography and the evolution of hermaphroditism. BMC Evol. Biol. 7: 104.

Poinar, G. O. 1972. Nematodes as facultative parasites of insects. Annu. Rev. Entomol. 17: 103-122.

Rasmann, S., et al. 2005. Recruitment of entomopathogenic nematodes by insect-damaged maize roots. Nature 434: 732-737.

Reinecke, A., Ruther, J., and Hilker, M. 2002. The scent of food and defence: green leaf volatiles and toluquinone as sex attractant mediate mate finding in the European cockchafer Melolontha melolontha. Ecol. Lett. 5: 257-263.

Rudel, D., and Sommer, R. J. 2003. The evolution of developmental mechanisms. Dev. Biol. 264: 15-37.

Ruther, J., Reinecke, A., Tolasch, T., and Hilker, M. 2001. Make love not war: a common arthropod defence compound as sex pheromone in the forest cockchafer Melolontha hippocastani. Oecologia 128: 44 47.

Ruther, J., Reinecke, A., and Hilker, M. 2002a. Plant volatiles in the sexual communication of Melolontha hippocastani: response towards timedependent bouquets and novel function of (Z)-3-hexen-1-ol as a sexual kairomone. Ecol. Entomol. 27: 76-83.

Ruther, J., Reinecke, A., Thiemann, K., Tolasch, T., Francke, W., and Hilker, M. 2000. Mate finding in the forest cockchafer, Melolontha hippocastani, mediated by volatiles from plants and females. Physiol. Entomol. 25: 172-179.

Ruther, J., Reinecke, A., Tolasch, T., and Hilker, M. 2002b. Phenolanother cockchafer attractant shared by Melolontha hippocastani Fabr. and M. melolontha L. Z. Naturforsch. C.-J. Biosci. 57: 910-913.

Schlager, B., Roseler, W., Zheng, M., Gutierrez, A., and Sommer, R. J. 2006. HAIRY-like transcription factors and the evolution of the nematode vulva equivalence group. Curr. Biol. 16: 1386-1394.

Shuto, Y., and Watanabe, H. 1987. Attractants from a vector, Monochamus alternatus, for the pine wood nematode. Agric. Biol. Chem. 51: 1457-1458. 
Stamps, W. T., and Linit, M. J. 1998. Chemotactic response of propagative and dispersal forms of the pinewood nematode Bursaphelenchus xylophilus to beetle and pine derived compounds. Fundam. Appl. Nematol. 21: 243-250.

Stamps, W. T., and Linit, M. J. 2001. Interaction of intrinsic and extrinsic chemical cues in the behaviour of Bursaphelenchus xylophilus (Aphelenchida: Aphelenchoididae) in relation to its beetle vectors. $\mathrm{Ne}$ matology 3: 295-301.
Schwenke, W. 1974. Die Forstschaedlinge Europas. Paul Parey, Hamburg. Van Valen, L. 1973. Festschrift. Science 180: 488.

West-Eberhard, M. J. 2005. Phenotypic accommodation: adaptive innovation due to developmental plasticity. J. Exp. Zool. B Mol. Dev. Evol. 304: 610-618.

Zheng, M., Messerschmidt, D., Jungblut, B., and Sommer, R. J. 2005. Conservation and diversification of Wnt signaling function during the evolution of nematode vulva development. Nat. Genet. 37: 300-304. 